\title{
A colorful view of the brain renin-angiotensin system
}

\author{
Curt D. Sigmund ${ }^{1} \cdot$ Justin L. Grobe ${ }^{1}$
}

Received: 9 December 2019 / Revised: 13 December 2019 / Accepted: 14 December 2019 / Published online: 17 January 2020

(C) The Japanese Society of Hypertension 2020

It is well established that the renin-angiotensin system (RAS) plays a crucial role in blood pressure, water and electrolyte balance, and metabolic (energy) homeostasis. The RAS is a classic endocrine system in which bloodborne angiotensin-II (Ang-II) acts on the Ang-II-receptorcontaining cells in various tissues to elicit its physiological effects. The observation that RAS inhibitors are effective antihypertensives even in patients with normal or low plasma renin activity has fueled the hypothesis that distinct, independently regulated RASs exist in a number of tissues [1]. There is substantial evidence for the presence of tissue RASs in the kidney, heart, adrenal gland, adipose, arterial wall, and brain, among others.

The brain is very responsive to Ang-II. Regions of the brain outside the blood-brain barrier (BBB), such as the circumventricular organs, may act as classic targets of endocrine Ang-II. Indeed, the presence of Ang-II receptors has been reported in the subfornical organ (SFO), organum vasculosum of the lamina terminalis (OVLT), and supraoptic nucleus, among other circumventricular organs [2]. Many of these regions control fluid and electrolyte homeostasis but also have connections with higher brain centers and nuclei controlling blood pressure. Other regions of the brain inside the BBB do not have access to bloodborne Ang-II and therefore cannot respond to classic endocrine Ang-II. De novo local synthesis of Ang-II has been proposed and reported [2]. The presence of Ang-II receptors in the paraventricular nucleus (PVN), amygdala, rostral ventrolateral medulla (RVLM) and nucleus tractus solitarius (NTS) suggests involvement in the baroreflex and sympathetic outflow. The localization of the Ang-II $\mathrm{AT}_{1} \mathrm{R}$ receptor on a subset of arcuate nucleus (ARC) neurons that are also positive for agouti-related peptide, along with

Curt D. Sigmund

csigmund@mcw.edu

1 Department of Physiology, Cardiovascular Center, Medical College of Wisconsin, Milwaukee, WI 53226, USA functional evidence, has suggested a role for the brain RAS in regulating resting metabolism and energy expenditure [3]. Ultimately, the physiological output of Ang-II in the brain depends on which Ang-II receptor is engaged, the cell type expressing the receptor (e.g., neuron vs astrocyte vs microglia), and in the case of neurons, the synaptic network engaged.

In the current issue of Hypertension Research, Sumners et al. [4] used mice carrying two separate reporter systems designed to identify cells expressing either Ang-II $\mathrm{AT}_{1} \mathrm{R}$ or $\mathrm{AT}_{2} \mathrm{R}$ or both. The primary rationale for the study was based on many observations that $\mathrm{AT}_{2} \mathrm{R}$ activation tends to oppose the actions of $\mathrm{AT}_{1} \mathrm{R}$ activation, and understanding the regulation of Ang-II-mediated output from the CNS requires an understanding of the relative cell types expressing or coexpressing each receptor under baseline conditions and in hypertension. The authors employed a dual reporter approach, in which the experimental readouts tdTomato and enhanced green fluorescent protein (eGFP) were used as surrogates for $\mathrm{AT}_{1} \mathrm{R}$ and $\mathrm{AT}_{2} \mathrm{R}$, respectively. The purpose of this editorial commentary is to ensure that the reader fully appreciates how these reporters are designed to function and what their results demonstrate.

The $\mathrm{AT}_{2} \mathrm{R}$ reporter used by Sumners et al. is a transgene consisting of a bacterial artificial chromosome (BAC) containing the $\mathrm{AT}_{2} \mathrm{R}$ gene, in which the eGFP gene was inserted in place of the $\mathrm{AT}_{2} \mathrm{R}$ coding region (Fig. 1a) [5]. Conceptually, eGFP should be expressed in cells that normally express $\mathrm{AT}_{2} \mathrm{R}$ without the expression of an additional (third) copy of the $\mathrm{AT}_{2} \mathrm{R}$ gene. There are numerous factors that will influence the readout of this type of reporter and thus its accuracy as a surrogate for $\mathrm{AT}_{2} \mathrm{R}$. First, the extent of regulatory (transcriptional) sequences must be sufficient to drive proper expression of eGFP as a representation of $\mathrm{AT}_{2} \mathrm{R}$-containing cells. In their original description of the transgene, they directly compared cells expressing eGFP (derived from the transgene) with cells expressing endogenous $\mathrm{AT}_{2} \mathrm{R}$ mRNA by in situ hybridization [5]. They provided evidence that $98 \%$ of the eGFP-positive cells were also positive for $\mathrm{AT}_{2} \mathrm{R}$ mRNA. Moreover, they showed that 
A

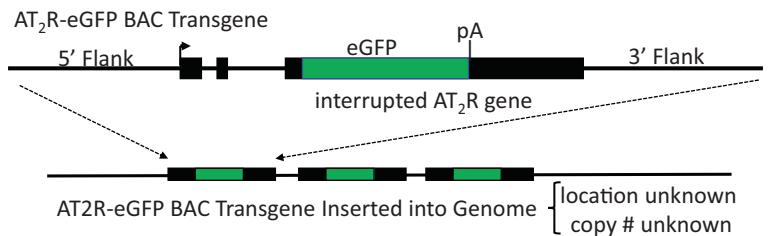

B

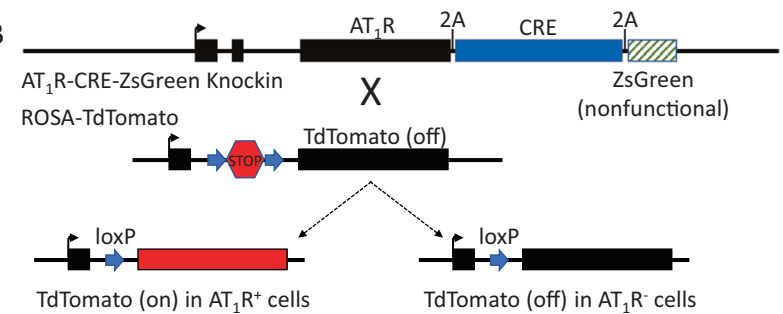

Fig. 1 Schematic representation of the reporter gene systems. a $A_{2} R-$ eGFP BAC Transgene: A BAC clone containing the $\mathrm{AT}_{2} \mathrm{R}$ gene was engineered to insert eGFP directly downstream of the initiation codon of the $\mathrm{AT}_{2} \mathrm{R}$ so that eGFP would be translated in place of $\mathrm{AT}_{2} \mathrm{R}$. The transgene is a GENSAT (Gene Expression Nervous System Atlas) product (http://www.gensat.org/index.html). Details on the transgene are also available from the Mutant Mouse Resources \& Research Center (MMRRC) under stock number 030278-UCD. The transgene integrated in an undetermined site in the genome, presumably in multiple copies, which may influence its level of expression. $\mathbf{b} \mathrm{AT}_{1} \mathrm{R}$ CRE-ZsGreen X ROSA-tdTomato: The Cre recombinase and ZsGreen genes were knocked into the $\mathrm{AT}_{1} \mathrm{R}$ in a manner that prevents the disruption of $\mathrm{AT}_{1} \mathrm{R}$. 2A sequences direct the translation of both genes, although ZsGreen was determined to be nonfunctional. When breed with ROSA-tdTomato reporter mice, the expression of Cre recombinase excises a loxP-STOP-loxP cassette that allows the expression and translation of tdTomato. Thus, any cells expressing Cre recombinase will express tdTomato, whereas cells that do not express Cre recombinase will not express tdTomato. As described in the text, the removal of the STOP cassette is permanent, which marks those cells and any daughter cells to be tdTomato positive. Any cell derived from $\mathrm{AT}_{1} \mathrm{R}$ positive cells, which at any time expressed Cre recombinase, will remain tdTomato positive even if the $\mathrm{AT}_{1} \mathrm{R}$ promoter is silenced

cells in at least one region of the brain that were devoid of eGFP staining were also negative for $\mathrm{AT}_{2} \mathrm{R}$ mRNA. The colocalization of $\mathrm{AT}_{2} \mathrm{R}$ mRNA and eGFP provides confidence in the fidelity of the reporter, at least under baseline unstimulated conditions. We can conclude with some confidence that the eGFP signals are unlikely to be false positives. Second, the site of insertion of the BAC transgene in the genome can influence how the transgene is expressed. Typically, large transgenes such as the one employed exhibit few position effects and are said to be immune from position effects. Thus, we will not comment on this issue further. Third, BAC transgenes are often expressed at a level that is proportional to copy number. One beneficial outcome of this is that the signal intensity can be artificially amplified if the copy number is high and thus false negatives can be diminished. This can be very advantageous when studying a gene that is expressed at very low levels, such as $\mathrm{G}$ protein coupled receptors or renin [6]. Based on the evidence presented, we can conclude that the eGFP readout in $\mathrm{AT}_{2} \mathrm{R}$-eGFP mice provides a reasonable contemporaneous signal for cells that express $\mathrm{AT}_{2} \mathrm{R}$. However, readers should approach each use of a reporter with some caution as it is incumbent on the authors to prove that their reporter provides an accurate readout of their specific target.

The $\mathrm{AT}_{1} \mathrm{R}$ reporter is more complicated as it consists of two separate genetic components (Fig. 1b). The first component is a knock-in mouse termed $\mathrm{AT}_{1 \mathrm{a}} \mathrm{R}$-Cre-ZsGreen, where Cre recombinase and the ZsGreen reporter are linked together (with 2a sequences) and knocked into the $\mathrm{AT}_{1} \mathrm{R}$ locus (at its normal location in the genome) [7]. Importantly, this was performed in a manner that did not disrupt $\mathrm{AT}_{1} \mathrm{R}$ expression. The authors noted that although the transgene was designed to simultaneously express both Cre recombinase and ZsGreen, they were unable to obtain evidence of ZsGreen expression by either fluorescence or immunohistochemistry. The reason for this failure to detect ZsGreen remains unclear and for the purpose of this commentary will be ignored. To detect Cre recombinase activity, the $\mathrm{AT}_{1 \mathrm{a}} \mathrm{R}-\mathrm{Cre}-\mathrm{ZsGreen}$ mice were bred with ROSAtdTomato mice, the second genetic component, which conditionally expresses tdTomato in response to the Cremediated removal of a loxP-STOP-loxP cassette. The loxPSTOP-loxP cassette prevents tdTomato expression when it is present. It is important for the reader to appreciate, as discussed by the authors, that this transgene will permanently mark any cell that in its developmental history expressed $\mathrm{AT}_{1} \mathrm{R}$ or activated the $\mathrm{AT}_{1} \mathrm{R}$ promoter. The reason for this is that Cre recombinase deletes the loxP-STOPloxP cassette permanently at the level of genomic DNA, and this genomic modification will be transmitted to any daughter cells after cell division. The most important concept to note is that the presence of a tdTomato signal does not necessarily signify the presence of concurrent $A_{1} R$ expression. Thus, a cell that is the daughter of an $\mathrm{AT}_{1} \mathrm{R}$ positive neuron but no longer expresses $A_{1} R$ itself will appear positive. This represents a limitation of this type of approach that can be overcome by contemporaneous detection of $\mathrm{AT}_{1} \mathrm{R}$ mRNA (by in situ hybridization) or protein (by immunohistochemistry). Of course, one of the main rationales for this study is the absence of antisera that have the specificity and selectivity to reliably detect these receptors [8]. However, although this limitation of the model may increase false-positive results, the permanent and strong activation of the tdTomato reporter gene in cells that have exhibited $\mathrm{AT}_{1} \mathrm{R}$ promoter activity greatly reduces the likelihood of false-negative results. In other words, while the interpretation of the concurrent expression levels of $\mathrm{AT}_{1} \mathrm{R}$ in tdTomato-positive cells is tenuous, investigators can conclude with increased confidence that the $\mathrm{AT}_{1} \mathrm{R}$ promoter was never activated in cells that do not exhibit tdTomato reporter expression. 
When both the $\mathrm{AT}_{1} \mathrm{R}$ and $\mathrm{AT}_{2} \mathrm{R}$ reporters are combined in a single animal (a triple transgenic mouse) as described in the accompanying article, the expression of eGFP is designed to identify cells that concurrently express $A_{2} R$, and the expression of tdTomato identifies cells that either concurrently express or previously expressed $\mathrm{AT}_{1} \mathrm{R}$. The reader must appreciate that the main readout of this study is entirely based on the transcriptional activity of the $\mathrm{AT}_{1} \mathrm{R}$ and $\mathrm{AT}_{2} \mathrm{R}$ promoters/regulatory regions. Importantly, the experiment provides no information regarding the bioavailability (translation, cell surface localization, and posttranslational decorations), subcellular localization (cell surface vs organelles, including nuclear and mitochondrial membranes), function (at the cellular, tissue, and organism levels), regulation (possible dimerization, cycling, GRK, $\beta$ arrestin, etc.), and second-messenger signaling of these receptors. Indeed, this study does not provide any correlation between neuroanatomical location and function. Therefore, the importance of previous work by the article's authors and many others using complementary methods (binding studies, genetic manipulations) should not be overlooked.

Therefore, what can we reliably conclude from the current study? First, $\mathrm{AT}_{1} \mathrm{R}$ and $\mathrm{AT}_{2} \mathrm{R}$ are generally expressed in different populations of neurons, although some $1-4 \%$ of neurons appear to have the capacity to express both receptors. That so few cells were observed to express both reporters provide support for the carefully stated conclusion that $\mathrm{AT}_{2} \mathrm{R}$ is not currently expressed in cells that have ever expressed $A T_{1} R$. $A T_{1} R$ was found to be expressed in the SFO, OVLT, PVN, median preoptic nucleus (MnPO), area postrema (AP), and NTS, all structures that are important for cardiovascular function, fluid balance, and metabolism. The relative content of $\mathrm{AT}_{1} \mathrm{R}$-positive neurons was greater than that of $\mathrm{AT}_{2} \mathrm{R}$-positive neurons in the OVLT, SFO, $\mathrm{PVN}$, and $\mathrm{ARC}$, whereas $\mathrm{AT}_{2} \mathrm{R}$-positive neurons predominated in the NTS and AP. For the most part, there were few differences in the number of cells expressing either receptor in males and females. Second, there is no clear evidence of $\mathrm{AT}_{1} \mathrm{R}$ or $\mathrm{AT}_{2} \mathrm{R}$ expression in microglia or astrocytes in response to DOCA-salt hypertension. In other words, there was no overlap in reporter fluorescence with Iba-1, a marker of microglia, or GFAP, a marker of astrocytes. Detailed data were provided for the PVN (for $\mathrm{AT}_{1} \mathrm{R}$ ) and NTS (for $\mathrm{AT}_{2} \mathrm{R}$ ), but the authors reported similar results (as data not shown) in the MnPO, SFO, and AP. This result was confirmed in the PVN of spontaneously hypertensive rats using RNAscope-based in situ hybridization methods to detect $\mathrm{AT}_{1} \mathrm{R}$ mRNA.
In summary, this study by Sumners et al. supports the conclusion that $\mathrm{AT}_{2} \mathrm{R}$ is rarely expressed in cells of the adult brain that have ever expressed $\mathrm{AT}_{1} \mathrm{R}$ and that the expression of both receptors appears to be tightly limited to neurons and not microglia or astrocytes. Further studies are needed to assess the functional characteristics and differences among $\mathrm{AT}_{1} \mathrm{R}$ - or $\mathrm{AT}_{2} \mathrm{R}$-positive neurons and the specific role of $\mathrm{AT}_{1} \mathrm{R}$ or $\mathrm{AT}_{2} \mathrm{R}$ in these neurons.

Funding This work was supported through research grants from the National Institutes of Health to CDS (HL084207, HL144807) and JLG (HL134850) and the American Heart Association to CDS (15SFRN23480000) and JLG (18EIA33890055).

\section{Compliance with ethical standards}

Conflict of interest The authors declare that they have no conflict of interest.

Publisher's note Springer Nature remains neutral with regard to jurisdictional claims in published maps and institutional affiliations.

\section{References}

1. Lavoie JL, Sigmund CD. Minireview: overview of the reninangiotensin system-an endocrine and paracrine system. Endocrinology. 2003;144:2179-83.

2. Forrester SJ, Booz GW, Sigmund CD, Coffman TM, Kawai T, Rizzo V, et al. Angiotensin II signal transduction: an update on mechanisms of physiology and pathophysiology. Physiol Rev. 2018;98:1627-738.

3. Claflin KE, Sandgren JA, Lambertz AM, Weidemann BJ, Littlejohn NK, Burnett CM, et al. Angiotensin AT1A receptors on leptin receptor-expressing cells control resting metabolism. J Clin Investig. 2017;127:1414-24.

4. Sumners C, Alleyne A, Rodrigues V, Pioquinto DJ, Ludin JA, Kar S, et al. Brain angiotensin type- 1 and type- 2 receptors: cellular location under normal and hypertensive conditions. Hypertens Res. 2020. https://doi.org/10.1038/s41440-019-0374-8 (e-pub ahead of print).

5. de Kloet AD, Wang L, Ludin JA, Smith JA, Pioquinto DJ, Hiller H, et al. Reporter mouse strain provides a novel look at angiotensin type-2 receptor distribution in the central nervous system. Brain Struct Funct. 2016;221:891-912.

6. Sinn PL, Davis DR, Sigmund CD. Highly regulated cell-type restricted expression of human renin in mice containing $140 \mathrm{~Kb}$ or $160 \mathrm{~Kb}$ P1 phage artificial chromosome transgenes. J Biol Chem. 1999;274:35785-93.

7. de Kloet AD, Wang L, Pitra S, Hiller H, Smith JA, Tan Y, et al. A unique "angiotensin-sensitive" neuronal population coordinates neuroendocrine, cardiovascular, and behavioral responses to stress. J Neurosci. 2017;37:3478-90.

8. Herrera M, Sparks MA, Alfonso-Pecchio AR, Harrison-Bernard LM, Coffman TM. Lack of specificity of commercial antibodies leads to misidentification of angiotensin type 1 receptor protein. Hypertension. 2013;61:253-8. 